\title{
Test, Treat, Track, Test, and Treat Active Surveillance toward Elimination of Schistosomiasis: A Feasibility Study
} Reda M. R. Ramzy, ${ }^{1 *}$ Amal Rabiee, ${ }^{2}$ Khaled M. Abd Elaziz, ${ }^{3}$ Carl H. Campbell Jr., ${ }^{4}$ Nupur Kittur, ${ }^{4}$ Daniel G. Colley, ${ }^{4,5}$ and
Ayat A. Haggag

${ }^{1}$ National Nutrition Institute, General Organization for Teaching Hospitals and Institutes, Cairo, Egypt; ${ }^{2}$ Ministry of Health and Population, Cairo, Egypt; ${ }^{3}$ Department of Community, Environmental and Occupational Medicine, Faculty of Medicine, Ain Shams University, Cairo, Egypt; ${ }^{4}$ Center for Tropical and Emerging Global Diseases, University of Georgia, Athens, Georgia; ${ }^{5}$ Department of Microbiology, University of Georgia, Athens,

Georgia

\begin{abstract}
We assessed the feasibility of using a test, treat, track, test, and treat (5T) active surveillance strategy to identify and treat individuals with schistosomiasis in three very low-prevalence villages in Kafr El Sheikh Governorate, Egypt. Primary index cases (PICs) were identified using the point-of-care circulating cathodic antigen (POC-CCA) assay in schools, in rural health units (retesting individuals with positive Kato-Katz examinations over the previous 6 months), and at potential water transmission sites identified by PICs and field observations. Primary cases identified potential secondgeneration cases-people with whom they shared water activities-who were then tracked, tested, and treated if infected. Those sharing water activities with second-generation cases were also tested. The yield of PICs from the three venues were 128 of 3,576 schoolchildren (3.6\%), 42 of 696 in rural health units (6.0\%), and 83 of 1,156 at water contact sites (7.2\%). There were 118 second- and 19 third-generation cases identified. Persons testing positive were treated with praziquantel. Of 388 persons treated, $368(94.8 \%)$ had posttreatment POC-CCA tests 3-4 weeks after treatment, and $81.8 \%$ (301) became negative. The 67 persons remaining positive had negative results after a second treatment. Therefore, all those found positive, treated, and followed up were negative following one or two treatments. Analysis of efforts as expressed in person-hours indicates that 4,459 person-hours were required for these $5 \mathrm{~T}$ activities, with nearly $65 \%$ of that time spent carrying out interviews, treatments, and evaluations following treatment. The 5T strategy appears feasible and acceptable as programs move toward elimination.
\end{abstract}

\section{INTRODUCTION}

Human schistosomiasis or Bilharzia is a neglected tropical disease (NTD) caused by blood flukes of the genus Schistosoma, currently endemic in 76 countries worldwide and has infected approximately 229.2 million people in $2018 .{ }^{1}$ Two forms of the disease are found in Africa: Schistosoma haematobium causes the urogenital form of the disease and Schistosoma mansoni causes the intestinal form of the disease. Infected people are mainly school-age children in rural areas as well as workers (farmers and fishermen) in contact with freshwater bodies infested with intermediate host snails. ${ }^{2}$

The WHO recommends preventive chemotherapy using anthelmintic drugs, either alone or in combination, as a public health measure for control/elimination of schistosomiasis in humans. Preventive chemotherapy can be implemented in three ways: 1) mass drug administration (MDA) when the whole population of a predefined geographical area is treated irrespective of clinical status, 2) targeted chemotherapy when only specific risk groups are treated; and 3) selective chemotherapy which screens patients and subsequently treats according to clinical status. ${ }^{3}$ Implementation of intensive control measures for several decades has resulted in significant reduction in the prevalence and morbidity of schistosomiasis in countries where the disease was highly endemic in the past, including China, Brazil, and Egypt. ${ }^{4}$ As shown in parts of Egypt and China, if carried out for decades, control interventions based on MDA can achieve very low levels of prevalence. At that point, MDA cost per case will be high, and

\footnotetext{
* Address correspondence to Reda M. R. Ramzy, National Nutrition Institute, General Organization for Teaching Hospitals and Institutes, 16 Kasr El Aini St., Cairo 11441, Egypt. E-mail: reda.mr.ramzy@ gmail.com
}

at least $90 \%$ of people treated will be uninfected, leading to massive overuse of drugs, and resources, and treatment fatigue.

Selective chemotherapy or testing, treatment, and surveillance provide a more cost-effective approach for chasing low numbers of remaining infected cases. For such an approach to be successful, every suspected $S$. mansoni case should be tested using a reliable rapid test and confirmed cases should be treated using praziquantel (PZQ). Then, the disease should be tracked through active epidemiological surveillance. Note that in 2012, the WHO initiated a comparable malaria "T3": test, treat, and track" as a useful tool for eliminating malaria deaths and eventually eradicating the disease. ${ }^{5}$

In this study, we evaluated the feasibility and effectiveness of an alternative approach for MDA in three communities, with very low S. mansoni prevalence in the Nile Delta region of Egypt. This strategy, termed as the "test, treat, track, test, and treat" (5T), is based on testing for S. mansoni infection using the urine-based point-of-care circulating cathodic antigen (POC-CCA) test, and treatment of confirmed cases using PZQ. They were tracked, tested with another POCCCA assay after 3-4 weeks, and retreated, if found POCCCA positive.

\section{MATERIALS AND METHODS}

Ethics statement. The Ethics Review Committee of the Ministry of Health and Population reviewed and approved the study protocol (May 18, 2019 dated March 21, 2019). Children were enrolled in the study after obtaining informed consent from their parents/guardians. Likewise, all enrolled adults provided informed consent. The work included only noninvasive collections of urine specimens. Providing a urine sample was taken as a child's assent. 
Study area. The study was conducted in three villages each located in Desouk, Al Riad or Sidy Salem district, Kafr El Sheikh Governorate. Each village has a population of approximately 6,000 people. The study villages were selected based on records in files at the Ministry of Health and population indicating low to very low $S$. mansoni prevalence ( $\approx 10 \%$ by POC-CCA assay).

Study participants. Schoolchildren (6-14 years), attending three schools in the study villages, were included in this study. All POC-CCA assay-positive subjects (scored as $1+, 2+$, and $3+)$, identified during the school survey, were included in the study as primary index cases (PICs). Because we have previously shown that in areas of very low prevalence, trace and sometimes $1+$ band readings of POC-CCA are likely to represent false-positive readings, ${ }^{6}$ subjects with POC-CCA scored as $1+, 2+$, and $3+$ were considered infected in the current work. This level of the POC-CCA reading $(1+$ and above) was decided as the positive cutoff for this study because the object of a 5T approach is to identify as many of those who are likely to be positive, so they can be treated and tracked. Furthermore, schistosomiasis-infected subjects recorded in the rural health units, based on Kato-Katz stool examination, during the 6 months before this study (October 2018-March 2019), were examined by POC-CCA assay, and all positives were included as PICs. In addition, POC-CCA assay-positive individuals, identified during direct observations of water contact sites, were included in the study as PICs.

All identified PICs were interviewed using a validated questionnaire to determine where they had contact with water and to determine the other individuals who frequented those potential water contact sites. Accordingly, individuals sharing water activities with PICs were identified and examined for schistosomiasis by using the POC-CCA assay. All POC-CCA assay-positive subjects (scored as $1+, 2+$, and $3+$ ) were included in the study as secondary index cases (SICs). Similarly, SICs were interviewed to identify other individuals sharing water activities with them, and POC-CCA assay-positive individuals from the latter group were included as the secondgeneration of SICs.

Assessment of water contact sites. Potential water contact sites were identified based on interviews with PICs and SICs, as well as by direct observations of individuals who then participated in the study. Assessments of potential water contact sites were carried out during the last week (i.e., 7 days) of each month during March-June 2019. For each potential water contact site, direct observation was carried out in two shifts, from 7 AM to 12.30 PM and from 12.30 PM to 5-6 PM. For each potential water contact site, the number of individuals entering the water, those contacting the water site for the first time, and the type of water activity were recorded. At the end of each observational week, recorded individuals were approached and asked to participate in the study. Those who consented (for children, consent was sought from parents or guardians at their homes), were assigned a coding number and enrolled in the study.

Point-of-care circulating cathodic antigen assay. The POC-CCA test (batch number 181116119, Ex. 11/2020), was performed according to the manufacturer's instruction (Rapid Medical Diagnostics, Pretoria, South Africa). In reading the POC-CCA results, we used grades $1+$ (intensity of the reactive band equal to the control band), $2+$, and $3+$ for scoring positive reactions. In this setting, trace reactions (fainter than the control band) was considered negative. The POC-CCA test was performed in three venues: primary schools, rural health units, and potential water contact sites.

Treatment of infected participants. All subjects with positive POC-CCA test results were treated using a standard $P Z Q$ dose (directly observed treatment with a single dose of 40 $\mathrm{mg} / \mathrm{kg}$ body weight). They were retested about 3-4 weeks after their initial $P Z Q$ dose and retreated, if they remained POC-CCA positive (not cured).

Recording information about the $5 \mathrm{~T}$ process. Recorded information about the 5T process included the time taken for various steps, the level of difficulty of each step, cost of local transportation, suggestions for increasing efficiency, yield in terms of individuals tested, and individuals testing positive using various approaches to identify potentially infected individuals, and barriers to implementing the test and treat strategy. These data were recorded on validated questionnaires by technicians and health workers involved in the study. For example, the level of difficulty of each step was reported using a scale of 1-5 (1, very easy; 2 , easy; 3 , moderate; 4 , difficult; and 5 , very difficult).

Statistical analysis. Data were checked for completeness and consistency. Data entry was carried out on Microsoft Excel database spreadsheet. Quantitative data were summarized by mean and SD, whereas qualitative data were summarized by frequencies and percentages. Data analysis was calculated with IBM SPSS statistics for Windows version 23 (IBM Corp., Armonk, NY). The chi-square test was used in analysis of this study. A " $P$-value" of less than 0.05 was considered statistically significant.

\section{RESULTS}

Identification of PICs by different venues. School survey. A total of 3,576 schoolchildren (6-15 years) were examined in the three villages of the study (Table 1, Figure 1). Of these, 1,753 children (49\%) and 1,823 children (51\%) were males and females, respectively. Of the schoolchildren tested, 3.6\% ( $n=$ 128) were POC-CCA positive and included as PICs (Figure 1). The school survey revealed that the percentage of identifying PICs in Desouk (1.6\%) was lower than that in AI Riad $(5.1 \%)$ and Sidy Salem (4.4\%) villages $\left(X^{2}=22.8, P=0.001 ; X^{2}=17.2\right.$, $P=0.001$, respectively).

Rural health units. Throughout the study, the rural health unit in Al Riad was under renovation, and so this part of the study was not conducted in this village. During the study period, 696 subjects were referred to the rural health units in the other two villages (Desouk and Sidy Salem villages) for examination of S. mansoni infection (Table 1, Figure 1). Of these, 381 subjects $(54.7 \%)$ were from Desouk village, their age ranged between 4 and 66 (mean \pm SD; $35.9 \pm 13.9$ ) years, and 224 (58.8\%) were males and 157 (41.2\%) females. Whereas, 315 subjects $(45.3 \%)$ were referred to the rural health unit in Sidy Salem village, their age ranged between 6 and $76(27.6 \pm 15.7)$ years, and 195 (61.9\%) were males and 120 (38.1\%) females. Overall, 42 subjects (6\%) were POC-CCA positive in rural health units and included as PICs (Figure 1). There was no difference in identifying PICs between the two villages.

Potential water contact sites. Assessment of the potential water contact sites revealed that 1,156 participants were in contact with water at least one time and were POC-CCA 
TABLE 1

Number of PICs as determined in three different case detection venues in low-prevalence Schistosoma mansoni areas in Egypt

\begin{tabular}{|c|c|c|c|c|c|c|}
\hline \multirow[b]{2}{*}{ Study site } & \multicolumn{2}{|c|}{ School survey (children aged 6-15 years) } & \multicolumn{2}{|c|}{ Screening at rural health units } & \multicolumn{2}{|c|}{$\begin{array}{l}\text { Surveillance at potential water } \\
\text { contact sites }\end{array}$} \\
\hline & Examined, $n$ & PICs, ${ }^{*} n(\%)$ & Examined, $n$ & PICs, $n$ (\%) & Examined, $n$ & PICs, $† n(\%)$ \\
\hline Desouk & 1,280 & $20(1.6)$ & 381 & $25(6.6)$ & 393 & $26(6.6)$ \\
\hline Al Riad & 1,021 & $52(5.1)$ & - & - & 413 & $19(4.6)$ \\
\hline Sidy Salem & 1,275 & $56(4.4)$ & 315 & $17(5.4)$ & 350 & 38 (10.9) \\
\hline Total & 3,576 & $128(3.6)$ & 696 & $42(6.0)$ & 1,156 & $83(7.2)$ \\
\hline
\end{tabular}

PIC = primary index cases.

${ }^{*}$ Number of PICs identified in Desouk schools was lower than that identified in Al Riad and Sidy Salem villages $\left(X^{2}=22.8, P=0.001 ; X^{2}=17.2, P=0.001\right.$, respectively).

† Number of PICs identified in Sidy Salem potential water contact site was significantly higher than that identified in Desouk and Al Riad villages $\left(X^{2}=4.3, P=0.03\right.$ and $X^{2}=10.8, P=0.001$, respectively).

tested on their first contact. Of these, 393 (34.0\%), 413 (35.7\%), and 350 (30.3\%) were from Desouk, Al Riad, and Sidy Salem villages, respectively (Table 1). Overall, 83 subjects (7.2\%) were POC-CCA positive in assessment of potential water contact sites and included as PICs (Figure 1). Assessment of potential water contact sites revealed that the percentage of identifying PICs was significantly higher in Sidy Salem (10.9\%) than that in Desouk (6.6\%) and AI Riad (4.6\%) villages $\left(X^{2}=4.3, P=0.03\right.$ and $X^{2}=10.8, P=0.001$, respectively).

In general, the proportion of PICs identified as a result of the school survey (3.6\%) was significantly lower than the ratio of identifying PICs at rural health units $(6 \%)\left(X^{2}=8.7, P=0.003\right)$ and potential water contact sites $(7.2 \%)\left(X^{2}=30.9, P=\right.$ 0.0001).

Identification of SICs and second-generation SICs by different venues. Of the initially tested 5,428 , the study identified a total of 390 infected subjects (7.2\%), categorized as PICs (64.8\%), SICs (30.3\%), and second-generation SICs (4.9\%). Their age ranged between 4 and $70(26.6 \pm 16.9)$ years, and $270(69.2 \%)$ were males and $120(30.8 \%)$ females. They included 95 subjects (24.4\%) from Desouk village, 93 (23.8\%) from Al Riad, and 202 (51.8\%) from Sidy Salem village (Figure 2). The POC-CCA readings ranged between 1+ (254 subjects; $65.1 \%$ ), 2+ (102 subjects; $26.2 \%$ ), and $3+$ (34 subjects; $8.7 \%$ ). The percentage of PICs and SICs identified in Sidy Salem was significantly higher than that in Desouk and $\mathrm{Al}$ Riad $\left(X^{2}=11.5, P=0.0007 ; X^{2}=42.7, P=0.0001\right.$, respectively). In addition, the percentage of SIC secondgeneration identified in Sidy Salem was higher that than in $\mathrm{Al}$ $\operatorname{Riad}\left(X^{2}=4.4, P=0.03\right)$ but not than that in Desouk.

Assessment of potential water contact sites. Potential water contact sites were assessed over 1 week of each of 4 months of the study duration. In Desouk village, the main recorded activities were agriculture practices (irrigation, surface irrigation for rice seedlings, etc.) and fishing (46.2\%),

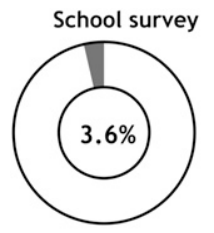

3,576 tested

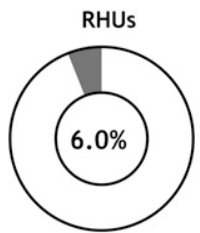

696 tested

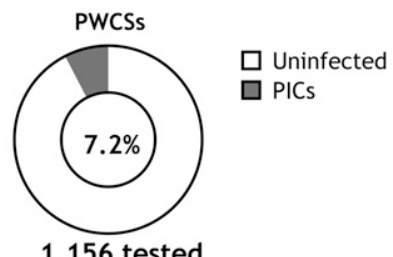

1,156 tested
FIGURE 1. Yield of primary index cases (PICs) as identified by three different case detection venues in low-prevalence Schistosoma mansoni areas in Egypt. PWCSs = potential water contact sites; $\mathrm{RHUs}=$ rural health units. washing utensils and clothes (39.2\%), and swimming and recreation (14.6\%). In Al Riad village, proportion recorded in these three activities was $69 \%, 15.5 \%$, and $15.5 \%$, respectively, whereas in Sidy Salem village, it was $70.6 \%$, $16.8 \%$, and $12.6 \%$, respectively.

Treatment of identified PICs and SICs. All subjects tested POC-CCA positive were offered treatment with a standard $P Z Q$ dose (directly observed treatment with a single dose of 40 $\mathrm{mg} / \mathrm{kg}$ body weight) and followed up after 3-4 weeks. Table 2 shows the data of PICs and SICs treatment with PZQ and follow-up with POC-CCA at 3-4 weeks after treatment. Of the 253 PICs, 251 subjects (99.2\%) were treated, although two individuals refused treatment. At the time of the follow-up, a lower percentage $(95.6 \%)(n=240)$ of treated subjects $\left(X^{2}=\right.$ $6.4, P=0.01$ ) were available for evaluation. In those evaluated, the cure rate reached $80.0 \%(n=192)$ and those who remained POC-CCA positive all turned negative (cured) after a second dose of PZQ. Similarly, $94.0 \%$ and $89.5 \%$ of the treated SICs and second-generation SICs were available for posttreatment follow-up evaluation, and the cure rate reached $85.6 \%$ and $82.3 \%$, respectively. The overall cure rate reached $81.7 \%$ after a single PZQ dose and $100 \%$ after a second dose. There was no difference in the cure rate (turning POC-CCA negative) between the three study categories.

Assessment of the 5T process. The overall efforts for pursuing the different $5 \mathrm{~T}$ tasks added up to 4,459 personhours. The least amount of effort (222 person-hours, 5\%) was spent at rural health units followed by efforts at schools $(605$

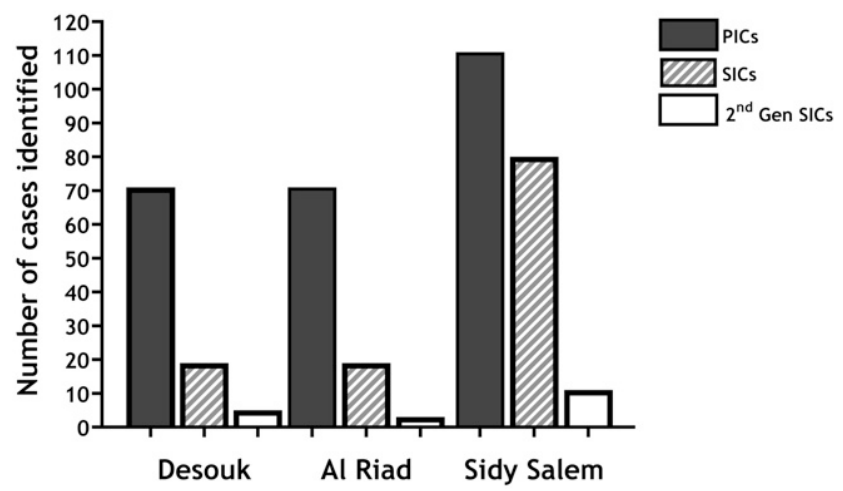

FIGURE 2. Number of primary index cases (PICs), secondary index cases (SICs) and second-generation SICs as presented by village. The proportion of PICs and SICs identified in Sidy Salem was significantly higher than that in Desouk and AI Riad $\left(X^{2}=11.5, P=0.0007 ; X^{2}=42.7\right.$, $P=0.0001$, respectively). The proportion of second-generation SICs identified in Sidy Salem was higher than that in Al Riad $\left(X^{2}=4.4, P=\right.$ 0.03) but not than that in Desouk. 
TABLE 2

Number of PICs and SICs treated with praziquantel and followed up with POC-CCA at 3-4 weeks after treatment

\begin{tabular}{|c|c|c|c|c|c|}
\hline \multirow[b]{2}{*}{ Category of cases } & \multirow[b]{2}{*}{ Identified, $n$} & \multirow[b]{2}{*}{ Treated, $n$} & \multicolumn{3}{|c|}{ Treatment outcome, $n(\%)$} \\
\hline & & & Followed up & Cured after single dose & Cured after two doses \\
\hline $\mathrm{PICs}$ & 253 & $251^{*}$ & $240(95.6)$ & $192(80.0)$ & $48(20.0)$ \\
\hline SICs & 118 & 118 & $111(94.0)$ & $95(85.6)$ & $16(14.4)$ \\
\hline Second-generation SICs & 19 & 19 & 17 (89.5) & 14 (82.3) & $3(17.7)$ \\
\hline Total & 390 & 388 & $368+(94.8)$ & $301(81.7)$ & 67 (18.3) \\
\hline
\end{tabular}

person-hours, $13.6 \%)$, and then 565 person-hours (12.7\%) for POC-CCA testing and interviews, followed by effort for assessment of potential water contact sites (804 person-hours, $18 \%)$, whereas the highest number of person-hours $(2,263$, $50.8 \%$ ) was needed for treatment and follow-up evaluations (Figure 3, Table 3). Among the study villages, there was no difference between the percentage of person-hours dedicated for activities at schools and at rural health units, assessment of potential water sites, and interviews and POC-CCA testing. However, the percentage of person-hours dedicated for treatment and follow-up evaluations was significantly higher in Sidy Salem villages that that in the other two study villages $\left(X^{2}=8.552 ; P=0.0035\right.$ and $X^{2}=8.024 ; P=$ 0.0046 , for Desouk and AI Riad, respectively).

The overall cost of transportation within the study area (study village and its surroundings) was estimated as US\$1,445.50. Of this, $29.1 \%$ (US\$422.00) was spent in Desouk village, $24.5 \%$ (US\$354.50) in Al Riad, and $46.3 \%$ (US\$669.00) in Sidy Salem village. The estimated cost of transportation was significantly higher in Sidy Salem village than Desouk $\left(X^{2}=90.974 ; P=0.0001\right)$ and Al Riad village $\left(X^{2}=\right.$ 150.094; $P=0.0001)$.

The level of difficulty of each step was reported by each of 24 persons (technicians and health workers) who were involved in conducting the study. On a scale of 1-5 (1 = very easy and $5=$ very difficult), the school survey was rated very easy and easy by $70.8 \%$ and $29.2 \%$, respectively. Working at rural health units, in Desouk and Sidy Salem combined, was rated very easy and easy by $75 \%$ and $25 \%$, respectively. Assessment of potential water contact sites was rated easy and difficult, each by $50 \%$ of the reporting persons. Treatment of infected subjects was rated very easy, easy, and moderate

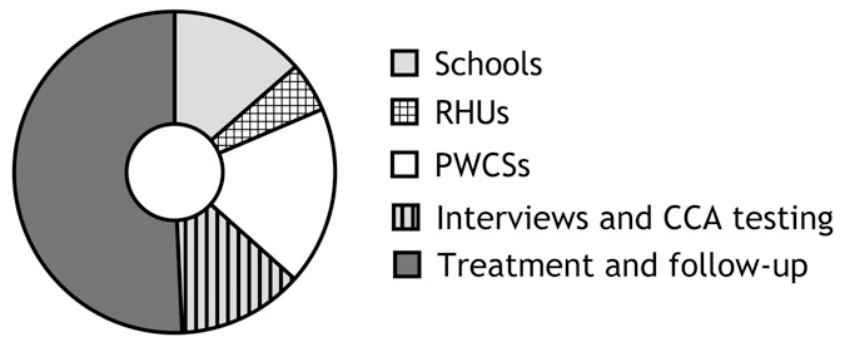

FIGURE 3. Percentage of person-hours spent in pursuing the test, treat, track, test, and treat strategy in three different case detection venues in low-prevalence Schistosoma mansoni areas in Egypt, including testing and interviews, treatment, and follow-up after treatment. Half the effort was for treating primary index cases and secondary index cases and follow-up evaluation. $\mathrm{RHUs}=$ rural health units; PWCSs = potential water contact sites. by $20.8 \%, 41.7 \%$, and $37.5 \%$, respectively. Posttreatment follow-up evaluation was rated moderate, difficult, and very difficult by $33.3 \%, 37.5 \%$, and $29.2 \%$, respectively.

\section{DISCUSSION}

The present study aimed to assess the feasibility of a new initiative T5: test, treat, track, test, and treat of human schistosomiasis mansoni. The strategy is based on active surveillance for identifying S. mansoni-infected cases through three venues: primary schools, rural health units, and potential water contact sites. Schistosoma mansoni infection was determined using the POC-CCA test, and subjects with POCCCA scored as $1+, 2+$, and $3+$ were considered infected in the current work. Infected cases were treated with a standard dose of PZQ and followed up with another POC-CCA assay after 3-4 weeks. The extensive active surveillance in the study villages, which had been under schistosomiasis control for many decades, confirmed their low S. mansoni prevalence ( $\leq 10 \%$ by POC-CCA assay), based on examination of schoolchildren, ${ }^{7}$ as the overall infection prevalence ranged between $3.6 \%$ (school survey) and $7.2 \%$ (assessment of potential water contact sites).

Comparison between the three study venues indicated that both the potential water contact site and rural health unit approaches identified larger numbers/percentages of S. mansoni infections than primary school surveys. This is probably because for many decades, schoolchildren in these schools had received annual mass treatment for schistosomiasis using standard dose of PZQ. In addition, people selfreferred to rural health units are routinely examined for schistosomiasis, and there is no charge for the diagnosis and treatment of schistosomiasis. Note that in Egypt, there is a well-developed network of rural health units, providing primary health care to major as well as satellite villages in the high-density Nile Delta and Nile valley, where almost everyone lives within $5 \mathrm{~km}$ of a rural health unit. ${ }^{8}$ Therefore, this approach could readily be used to apply the " $5 \mathrm{~T}$ " strategy in other S. mansoni-endemic governorates.

Assessment of potential water contact sites indicated that a considerable number of people (46.2-70.6\%) are in contact with potential transmission sites because of occupational activities such as farming in irrigated agricultural areas and fishing. Other water contact activities included domestic activities such as washing clothes and utensils (15.5-39.2\%) and recreational activities and swimming (12.6-15.5\%). Such observations could explain our finding that assessment of potential water contact sites resulted in identification of the largest number of PICs. Schistosomiasis is a mainly rural, 
TABLE 3

Efforts dedicated for pursuing different test, treat, track, test, and treat activities as expressed in person-hours

\begin{tabular}{|c|c|c|c|c|c|c|}
\hline \multirow[b]{2}{*}{ Study site } & \multicolumn{5}{|c|}{ Activity in person-hour (\%) } & \multirow[b]{2}{*}{ Total } \\
\hline & School survey & $\begin{array}{l}\text { Identification of primary index } \\
\text { cases at rural health units }\end{array}$ & $\begin{array}{l}\text { Assessment of potential } \\
\text { water sites }\end{array}$ & $\begin{array}{l}\text { Circulating cathodic antigen } \\
\text { testing and interviews }\end{array}$ & $\begin{array}{l}\text { Treatment and } \\
\text { follow-up }\end{array}$ & \\
\hline Desouk & $192(4.3)$ & $99(2.2)$ & $273(6.1)$ & $176(3.9)$ & 703 (15.8) & $1,443(32.4)$ \\
\hline Al Riad & $197(4.4)$ & - & 249 (5.6) & $177(4.0)$ & 710 (15.9) & $1,333(29.9)$ \\
\hline Sidy Salem & $216(4.8)$ & $123(2.8)$ & $282(6.3)$ & $212(4.8)$ & 850 (19.1) & $1,683(37.7)$ \\
\hline Total & 605 (13.6) & $222(5.0)$ & $804(18.0)$ & $565(12.7)$ & $2,263(50.8)$ & $4,459(100)$ \\
\hline
\end{tabular}

percentage of person-hours was spent to carry out interviews, treatment, and follow-up evaluation in Sidy Salem village than Desouk and AI Riad $(X 2=9.5, P=0.008)$ villages.

often occupational disease that principally affects people who are unable to avoid contact with water, regardless of the weather. ${ }^{9}$ Note that the current study was conducted during the months of March-June when the average temperature was $20.0^{\circ} \mathrm{C}\left(68.0^{\circ} \mathrm{F}\right)$. If the study had been carried out during warmer or hot months, we may have found more people in contact with potential water contact sites, especially for playing and swimming. Consequently, our study validates assessment of potential water contact sites as a potential approach for implementing the " $5 \mathrm{~T}$ " strategy in other similar endemic areas.

The WHO recommends MDA for control/elimination of several NTDs. ${ }^{3}$ However, cost-effectiveness and achieving and maintaining an optimal high MDA coverage will remain challenges for successful MDA campaigns because infected cases remain unknown. The $5 \mathrm{~T}$ strategy has prominent advantages over MDA, especially when the prevalence has decreased to low or very low levels. It targets infected individuals detected in the general population for treatment and directly approaches noncompliant individuals or those who are not commonly treated through MDA. In the present study, the compliance for treatment and follow-up after PZQ treatment was excellent, as $99.2 \%$ of the infected individuals were treated and $94.8 \%$ tracked for a posttreatment follow-up. In other types of longitudinal studies, lower compliance for follow-up after treatment was reported. For example, in two cohort studies, $82.6 \%$ and $60.7 \%$ of those enrolled were followed up 3-4 weeks ${ }^{10}$ and 1 year after PZQ treatment, respectively. ${ }^{11}$ Such high compliance with the follow-up after treatment could be because the follow-up was carried out after a relatively short period (3-4 weeks) posttreatment. Furthermore, the technicians and health workers who carried out the study are well known to the studied communities which would ensure high-level community participation.

In the present study, we used PZQ at the WHO-recommended dose $(40 \mathrm{mg} / \mathrm{kg})$ for treatment of infected subjects in all ages. Note that most of these infected subjects were previously treated with PZQ at least once. However, 14 treatment-naive children (age $\leq 6$ years) were treated for the first time. High cure rates (81.7\% after one dose and $100 \%$ after the second dose) were observed using a sensitive diagnostic test (POC-CCA). These are higher than cure rates (42-79\% after single dose and 69-91\% after two doses) reported by a review article based on positive to negative conversion in egg detection assays. ${ }^{12}$ It is of special interest that the POC-CCA trace reading was not observed in any of the posttreatment evaluations. ${ }^{6}$

Our observation that the number of identified infected subjects decreased as the number of detection cycles increased is of special interest. Initially, there were 253 PICs, which decreased to 118 SICs (after the second detection cycle), then decreased to 19 seconds SICs (after a third detection cycle). This observation is consistent for the three study villages. Considering that the study villages have a very low S. mansoni infection prevalence $(\leq 10 \%$ by POC-CCA assay), such an observation clearly indicates that these villages are approaching elimination of transmission.

Our findings of the assessment of the " $5 \mathrm{~T}$ " process indicate its feasibility. The process goals are achievable, acceptable to field technicians as well as village dwellers, and probably costeffective as programs move toward elimination. This approach is certainly cost-effective in regard to drug costs. Note that this is a limited study, and so there was no broad community mobilization. It is worth mentioning that we were not able to find any published formal study based on testing and treating of schistosomiasis with which we could compare of our findings. However, the " $5 \mathrm{~T}$ " as implemented in the present study is currently labor-intensive, and for widespread application, the strategy needs to be streamlined to reduce persontime requirements. For adopting and implementing this strategy on a wide scale by an elimination program, several important issues need to be resolved or fulfilled. First, strong political commitment of the Ministry of Health and Population senior officials, as well as local officials, is a crucial element for its success, as seen in other public health programs. ${ }^{13} \mathrm{Sec}-$ ond, good advocacy using different venues including advertising posters in schools, rural health units, and agricultural extension stations, and meetings with the chief and religious leaders of target villages would likely help maximize case detection yields. Third, extensive training of field-workers and adequate supervision are essential ingredients for success.

Received February 28, 2020. Accepted for publication June 5, 2020. Published online July 13, 2020.

Acknowledgments: We appreciate the communities of the study villages for their cooperation and participation in this study. We acknowledge the efforts of the 24 technicians and health workers led by Khaled Gad, chief technician, Endemic Diseases Department, Kafr El Sheikh Governorate, for their dedication and technical expertise. We also thank the Director of Endemic Diseases in Kafr El Sheikh Governorate for his support in implementing the study, and Global Health Development and the Eastern Mediterranean Public Health Network for their provision of logistical support in the conduct of the study.

Financial support: This study received financial support from the University of Georgia Research Foundation, Inc., which was funded by the Bill \& Melinda Gates Foundation for the SCORE project.

Authors' addresses: Reda M. R. Ramzy, National Nutrition Institute, General Organization for Teaching Hospitals and Institutes, Cairo, Egypt, E-mail: reda.mr.ramzy@gmail.com. Amal Rabiee and Ayat A. Haggag, Ministry of Health and Population, Cairo, Egypt, E-mails: amalrabiee1@gmail.com and ayatef@yahoo.com. Khaled M. Abd Elaziz, Department of Community, Environmental and Occupational Medicine, Faculty of Medicine, Ain Shams University, Cairo, Egypt, 
E-mail: khaledabdu@yahoo.com. Carl H. Campbell, Jr., Nupur Kittur, and Daniel G. Colley, Center for Tropical and Emerging Global Diseases, University of Georgia, Athens, GA, E-mails: ccamp@uga.edu, nkittur@uga.edu, and dcolley@uga.edu.

This is an open-access article distributed under the terms of the Creative Commons Attribution (CC-BY) License, which permits unrestricted use, distribution, and reproduction in any medium, provided the original author and source are credited.

\section{REFERENCES}

1. World Health Organization, 2019. Schistosomiasis and soil transmitted helminthiases: numbers of people treated in 2018. Weekly Epidemiological Rec 50: 601-612.

2. Colley DG, Bustinduy AL, Secor WE, King CH, 2014. Human schistosomiasis. Lancet 383: 2253-2264.

3. WHO, 2006. Preventive Chemotherapy in Human Helminthiasis: Coordinated Use of Anthelminthic Drugs in Control Interventions: A Manual for Health Professionals and Programme Managers. Geneva, Switzerland: World Health Organization.

4. WHO, 2013. Schistosomiasis: Progress Report 2001-2011, Strategic Plan - 2020. Geneva, Switzerland: World Health Organization

5. WHO, 2012. T3: Test. Treat. Track. Scaling up Diagnostic Testing, Treatment and Surveillance for Malaria. Geneva, Switzerland: World Health Organization.

6. Haggag AA, Casacuberta Partal M, Rabiee A, Abd Elaziz KM, Campbell CH, Colley DG, Ramzy RMR, 2019. Multiple praziquantel treatments of Schistosoma mansoni egg-negative, CCA-positive schoolchildren in a very low endemic setting in
Egypt do not consistently alter CCA results. Am J Trop Med Hyg 100: 1507-1511.

7. Haggag AA, Rabiee A, Abd Elaziz KM, Gabrielli AF, Abdel Hay R, Ramzy RMR, 2017. Mapping of Schistosoma mansoni in the Nile delta, Egypt: assessment of the prevalence by the circulating cathodic antigen urine assay. Acta Trop 167: 9-17.

8. el Katsha S, Watts S, 1995. Schistosomiasis control through rural health units. World Health Forum 16: 252-254.

9. Barakat R, Farghaly A, El Morshedy H, Hassan M, de Miller W, 1998. Impact of national schistosomiasis control program in Kafr El-Sheikh governorate, Nile delta, Egypt: an independent evaluation. J Egypt Public Health Assoc 73: 737-753.

10. Sousa-Figueiredo JC, Betson $M$, Atuhaire $A$, Arinaitwe $M$, Navaratnam AM, Kabatereine NB, Bickle Q, Stothard JR, 2012. Performance and safety of praziquantel for treatment of intestinal schistosomiasis in infants and preschool children. PLoS Negl Trop Dis 6: e1864.

11. Balen J, Stothard JR, Kabatereine NB, Tukahebwa EM, Kazibwe F, Whawell S, Webster JP, Utzinger J, Fenwick A, 2006. Morbidity due to Schistosoma mansoni: an epidemiological assessment of distended abdomen syndrome in Ugandan school children with observations before and 1-year after anthelminthic chemotherapy. Trans R Soc Trop Med Hyg 100: 1039-1048.

12. King CH, Olbrych SK, Soon M, Singer ME, Carter J, Colley DG, 2011. Utility of repeated praziquantel dosing in the treatment of schistosomiasis in high-risk communities in Africa: a systematic review. PLoS Negl Trop Dis 5: e1321.

13. Ramzy RMR, Kamal HA, Hassan MA, Haggag AA, 2019. Elimination of lymphatic filariasis as a public health problem from the Arab Republic of Egypt. Acta Trop 199: 105121. 